\title{
Análisis de factibilidad técnica para la producción de bioetanol a partir de residuos de maíz en Ecuador
}

\section{Technical feasibility analysis for the production of bioethanol from maize residues in Ecuador}

\author{
Paúl Hernández Rueda \\ Universidad Técnica del Norte, Ecuador \\ Carlos Mafla Yépez \\ Universidad Técnica del Norte, Ecuador \\ Ignacio Benavides Cevallos \\ Universidad Técnica del Norte, Ecuador \\ Fernando Ramírez Paredes \\ Universidad Técnica del Norte, Ecuador
}

Autor por Correspondencia: ephernandez@utn.edu.ec cnmafla@utn.edu.ec, ibbenavides@utn.edu.ec, frramirez@utn.edu.ec

Fecha de recepción: 19 de Enero de 2018 - Fecha de aceptación: 25 de julio de 2018

\section{Resumen}

En el presente trabajo se produjo bioetanol a partir de residuos de maíz en la provincia de Imbabura-Ecuador. Se ha utilizado los residuos del maíz específicamente del tipo "Zea mays 1" (Chaucho Mejorado) que se produce en la provincia de Imbabura.

Se determinó su factibilidad en cuanto a su posible producción en términos de biomasa disponible. Se ha caracterizado el etanol en una mezcla E10 es decir 10\% de etanol y $90 \%$ de Gasolina Súper, para determinar su factibilidad en su uso para el motor como combustible, teniendo resultados satisfactorios.

Palabras Claves: biomasa; etanol; biocombustible; maíz; desechos

\begin{abstract}
In the present work bioethanol was produced from corn residues in the province of ImbaburaEcuador. It has been used corn residues specifically of the type "Zea mays 1" (Improved Chaucho) that is produced in the province of Imbabura.

Its feasibility was determined in terms of its possible production in terms of available biomass. Ethanol has been characterized in an E10 mixture that is 10\% ethanol and $90 \%$ Super Gasoline, to determine its feasibility in its use for the engine as a fuel, having satisfactory results.
\end{abstract}

Key words: biomass; ethanol; biofuel; corn; waste 


\section{Introducción}

La problemática ambiental actual obliga a buscar los medios necesarios para mitigar el impacto ambiental causado por la continua emanación de gases de efecto invernadero (Naz, 2014). Estas emanaciones son una parte del resultado de la combustión, en el caso del mundo automotriz una solución utópica será el salto a los vehículos eléctricos. Ese salto representaría un costo infranqueable para la sociedad actual, razón por la cual se pretende desarrollar mecanismos de transición entre los vehículos con motor de combustión y los vehículos eléctricos. Una problemática adicional es la dependencia del petróleo que es un recurso no renovable, cuyo fin se estima dentro de los siguientes 50 años (OPEP, 2017).

Por este motivo los biocombustibles, al provenir de una fuente renovable de energía, representan una alternativa viable. Un beneficio adicional es que el bio etanol puede obtenerse aprovechando la biomasa residual, a esto se suma que existe un rango de mezcla entre biocombustible y combustible de origen fósil tolerable por los motores existentes sin modificaciones mecánicas. Es por estos motivos que la investigación en biocombustibles es una línea con un alto impacto a nivel mundial. (Elfasakhany, Volume 139)

\section{Materiales y métodos}

Para este proyecto se propone utilizar a la semilla de maíz que es distribuida a los agricultores de la provincia de Imbabura por parte del Ministerio de Agricultura y Ganadería (MAGAP). Con base en los datos proporcionados por este ministerio se conoce que la semilla que se distribuye es la "INIAP -122 Chaucho Mejorado", que corresponde a una variedad de maíz amarillo harinoso que se adapta de manera apropiada a las condiciones geográficas y climáticas de la provincia de Imbabura.

\section{Tabla 1. Ficha técnica del "Chaucho Mejorado"}

\begin{tabular}{ll}
\hline $\begin{array}{l}\text { Importantes características del "Chaucho } \\
\text { Mejorado" }\end{array}$ & \\
\hline $\begin{array}{l}\text { 1. Morfológicas y } \\
\text { Agronómicas }\end{array}$ & Promedio \\
$\begin{array}{l}\text { Díaz transcurridos hasta la floración femenina } \\
\text { (presencia de flor) }\end{array}$ & 102 \\
Díaz transcurridos a cosecha en choclo & 135 \\
Díaz transcurridos a cosecha en seco & 225 \\
Altura & $250 \mathrm{~cm}$ \\
Color de grano seco/ tierno & Amarillo/Crema \\
Maneras de consumirlo & Harina, Tostado, \\
& Choclo \\
2. Calidad (Seco) & Promedio \\
\hline \multicolumn{2}{c}{ (Yánez G., 2013) }
\end{tabular}




\section{Determinación de la disponibilidad de biomasa residual en Imbabura}

La materia prima que se necesita para llevar a cabo la producción de bioetanol se considera como potencialmente de fácil acceso en la provincia de Imbabura. Esto se debe a las buenas condiciones climáticas y geográficas que posee la provincia (Dias, 2011)

Tabla 2. Propiedades climáticas y geográficas de Imbabura

\begin{tabular}{ll}
\hline Altura & $2215 \mathrm{~m} \mathrm{~s} . \mathrm{n} . \mathrm{m}$. \\
Temperatura & $17^{\circ} \mathrm{C}$ \\
Humedad & $68 \%$ \\
Clima & Templado \\
Coordenadas & $0^{\circ} 21^{\prime} 00^{\prime \prime} \mathrm{N} 78^{\circ} 08^{\prime} 00^{\prime \prime} \mathrm{O}$ \\
Superficie & $4599 \mathrm{~km}^{2}$ \\
\hline \multicolumn{2}{c}{ Fuente: (Prefectura de Imbabura, 2016) }
\end{tabular}

Imbabura se encuentra conformada políticamente por seis cantones: Otavalo, San Miguel de Urcuquí, Antonio Ante, Cotacachi, Ibarra y Pimampiro, los mismos que cuentan con importantes áreas para la producción de maíz del tipo Chaucho Mejorado, a continuación, se muestra la distribución geográfica de ellos.

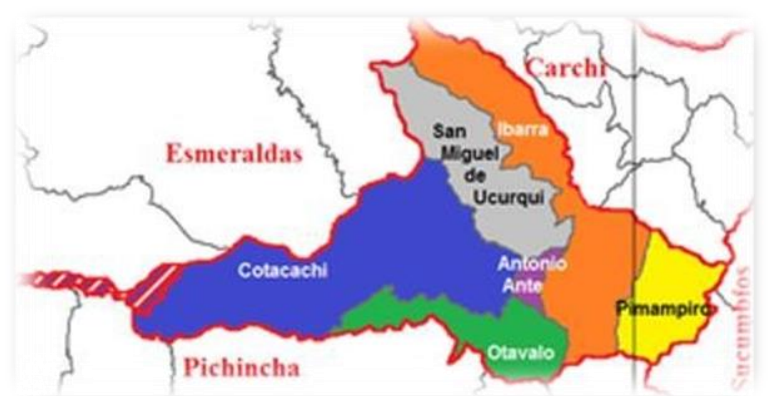

Figura 1. Distribución geográfica de Imbabura y sus cantones Fuente: (MAGAP-DPA UZI, 2016)

La Tabla 4 muestra la utilización de hectáreas cultivables dedicadas a la producción de maíz, por cada cantón de la provincia de Imbabura.

Tabla 3. Hectárea de Maíz Cosechadas en Imbabura en el 2016

\begin{tabular}{llc}
\hline Cantones & $\begin{array}{c}\text { Parroquia con mayor } \\
\text { producción }\end{array}$ & $\begin{array}{c}\text { Ha cosechadas } \\
\text { cantón }\end{array}$ \\
\hline Ha Antonio Ante & San Roque & 8 \\
Ha Cotacachi & Imantag & 603 \\
Ha Ibarra & Angochagua & 262 \\
Ha Otavalo & Gonzales Suarez & 808 \\
Ha Pimampiro & Pimampiro & 123 \\
Ha Urcuquí & Cahuasqui & 40 \\
Ha Totales & 1,844 & \\
\hline
\end{tabular}


Fuente: (MAGAP-DPA UZI, 2016).

\section{Método de obtención del etanol}

El Etanol se obtiene mediante un proceso de Hidrólisis. En este proceso una molécula de celulosa es dividida por la gestión de una molécula de agua. Todo se lleva a cabo con la ayuda de una enzima o de una bacteria. De este modo las estructuras de la celulosa, lignina y hemicelulosa se dividen consiguiendo una solución azucarada fermentable (Lucero, 2016).

Con la finalidad extraer los azucares almacenados en la biomasa residual del maíz se ha utilizado la Enzima D-Xilosa. Esta enzima es un monosacárido o azúcar proveniente de la madera, presente también en las paredes celulares de plantas (ver Tabla 6).

Tabla 4. Métodos para tratar la materia Orgánica

\begin{tabular}{ll}
\hline Evaluación de métodos para tratar la materia Orgánica \\
\hline Método & Tiempo de \\
& Obtención \\
Hongos xilófagos & 26 Díaz de \\
& Incubación \\
Enzimas (Xilosa) & Inmediato \\
\hline
\end{tabular}

Fuente: (Espinosa Cajas, 2013).

La enzima D-Xilosa rompe las paredes de las moléculas celulósicas de la biomasa residual del maíz, liberando los azucares almacenados. Posteriormente, esta biomasa modificada, en adición de levaduras, es fermentada y destilada obteniendo así el bioetanol (Sánchez Riaño \& Gutiérrez Morales, 2010)

Tabla 5. Ficha Técnica de Enzima D-Xilosa

\begin{tabular}{ll}
\hline Enzima D-Xilosa & \\
\hline Característica & Dato \\
Formula Molecular & C5 H10 O5 \\
Grado & Puris \\
Peso molecular & 150.13 \\
Duración & 5 años \\
Valor PH & $4.5-6.0$ \\
Color & Cristalino blanco \\
Densidad (g/cm3) & 1,525 \\
Olor & Inodoro \\
Estado físico a 20 C & Sólido \\
\hline \multicolumn{2}{c}{ Fuente: (MAGAP-DPA UZI, 2016). }
\end{tabular}




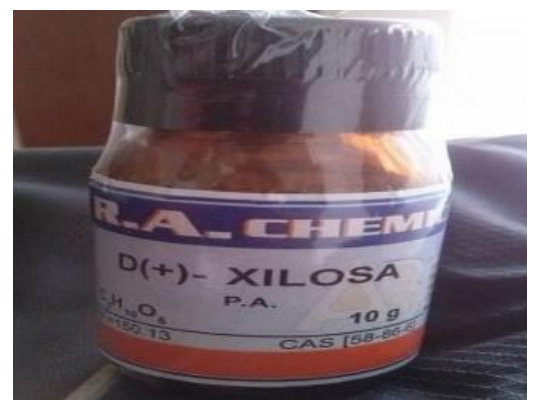

Figura 2. Enzima D-Xilosa.

Una vez conocidas las características del maíz a utilizar, las zonas productivas dedicadas al maíz y el método de tratamiento de la biomasa, es pertinente detallar el proceso para la producción del bioetanol.

Recolección de la materia prima. en este caso de la biomasa residual agrícola de maíz se realiza en uno de los sectores registrados como potencialmente productores de Biomasa, debido a la cercanía se la lleva a cabo en la ciudad de Otavalo, dicha recolección de materia prima tiene lugar en una zona delimitada de $10 \mathrm{~m}^{2}$, posteriormente de realizada la recolección de biomasa residual agrícola (tallos, hojas) se procede al triturado y pesado de las muestras obtenidas, es importante mencionar que dicho proceso se da lugar al constatar que el grado de humedad relativa de la materia prima es del $33 \%$

Una vez obtenida la materia prima seca, es necesario picarla o triturarla hasta que la misma tenga una longitud aproximada de 0.3 a $0.8 \mathrm{~cm}$, de esta manera la hidrolisis enzimática a realizarse sobre la materia prima.

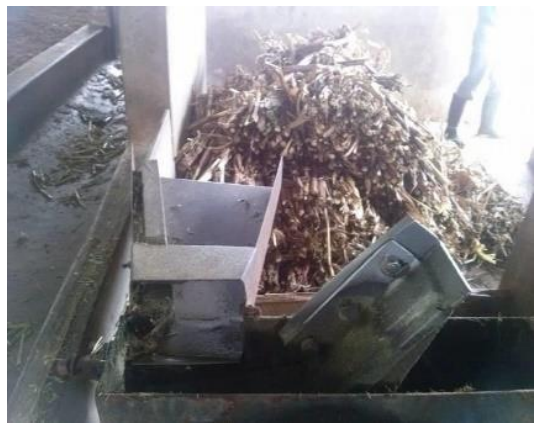

Figura 3. Materia prima triturada.

Pesaje de materia prima seca

Al completar el proceso anterior se puede disponer de la biomasa residual de maíz, es necesario pesarla para conocer sus características en condiciones secas $(33.03 \%$ de humedad relativa).

Tabla 6. Pesaje de Muestras Secas

\begin{tabular}{ll}
\hline Área $\left(\mathrm{m}^{2}\right)$ & Kg registrados \\
\hline 10 & $\mathbf{8 9 . 3 6}$ \\
\hline
\end{tabular}




\section{Tratamiento enzimático}

Como anteriormente se mencionó, para lograr romper las paredes moleculares de la biomasa residual agrícola de maíz y extraer los azucares almacenados en ella, se precisa la utilización de la enzima D- Xilosa.

Rompimiento de paredes moleculares y liberación de azucares fermentables

El proceso de la hidrólisis o rompimiento de las paredes de moleculares de la biomasa residual agrícola de maíz (celulosa) consiste básicamente en la añadidura de una molécula de agua a cada molécula de celulosa o materia prima transformándola en Glucosa o azucares fermentables disueltos. En este punto se coloca 11,36 Kg de materia prima triturada en un recipiente plástico, juntamente con:

- Enzima D- Xilosa 0,75 gr por cada Kg de materia prima

- L de agua a una temperatura de $60^{\circ} \mathrm{C}$

Tras colocar los antes mencionados elementos en el interior de un recipiente de plástico, se los dejará reposar durante 8 días, tiempo necesario para la actuación de la enzima sobre la materia prima, es necesario que la temperatura ambiente se encuentre en $30{ }^{\circ} \mathrm{C}$ de manera estable.

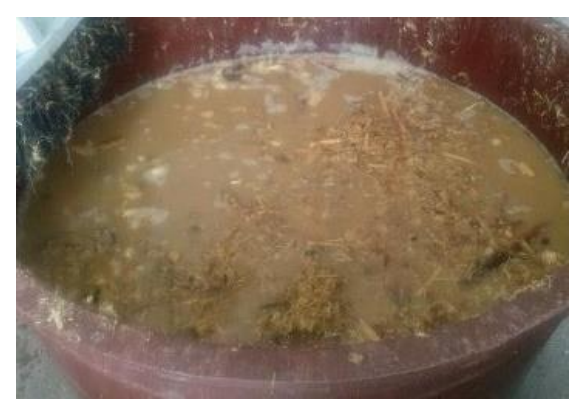

Figura 4. Materia prima triturada

Transcurridos 2 días de iniciado el proceso de hidrólisis es necesario controlar periódicamente la cantidad de azucares disueltos (Grados Brix) en la substancia mezclada inmersa en el recipiente de plástico, a continuación, se muestra el resultado de dicho control.

Tabla 7. Resultados de control

\begin{tabular}{ll}
\hline $\begin{array}{l}\text { Días } \\
\text { transcurridos }\end{array}$ & Grados Brix \\
\hline 2 & 2 \\
4 & 5.2 \\
5 & 8.5 \\
6 & 10 \\
7 & 11.5 \\
\hline
\end{tabular}




\section{3}

Al termino de 8 días después de iniciado el proceso enzimático y el posterior control de grados Brix disueltos en la mezcla, se tiene que los mismos son lo suficientemente altos para proceder a fermentarlos (grados Brix $\left.13^{\circ}\right)$.

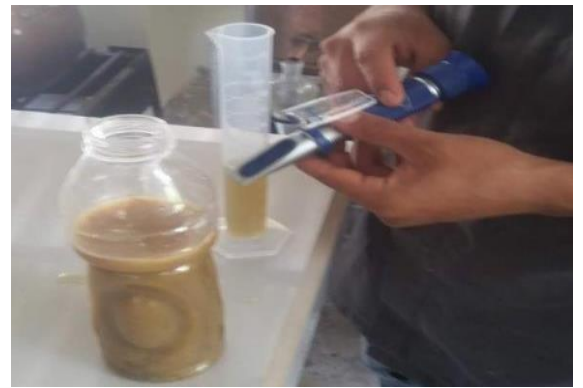

Figura 5. Control de Grados Brix.

\section{Fermentación}

Obtenidos los azúcares fermentables a partir del proceso anterior se procede a la fermentación aeróbica de la substancia resultante, para este proceso se procede a añadir a dicha substancia $0.68 \mathrm{Kg}$ de Levadura manteniendo la temperatura ambiente constante de $30{ }^{\circ} \mathrm{C}$.

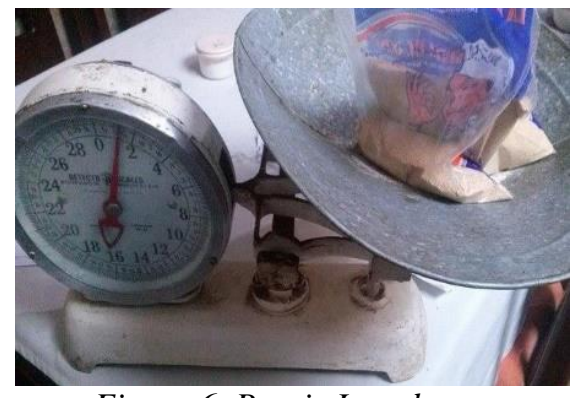

Figura 6. Pesaje Levadura

Tras la adición de $0.68 \mathrm{Kg}$ de levadura además del paso de 3 días posteriores se observa la presencia de burbujas en la substancia o mezcla dentro del recipiente del plástico, lo que nos indica la expulsión de $\mathrm{CO} 2$ por parte de la mezcla, además indicando que dicho elemento adicionado está actuando de manera favorable sobre la substancia.

Tras el transcurso de 8 días se observa que la presencia de burbujas en la mezcla desaparece y en su lugar existe una fina capa blanca de biomasa residual agrícola de maíz, mostrando de esta manera el consumo total del azúcar disuelto en la mezcla, pudiendo comprobárselo midiendo los grados Brix $\left(0^{\circ}\right)$. 


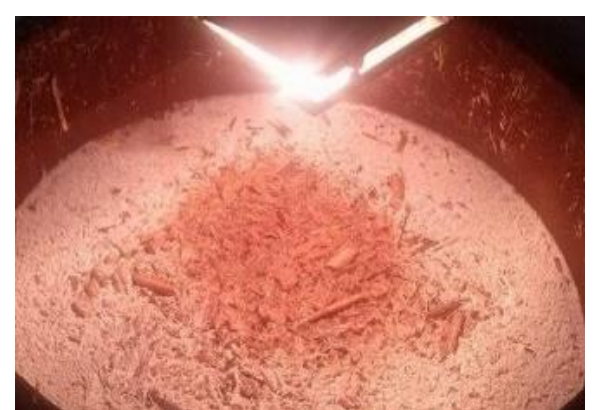

Figura 7. Presencia fina capa blanca en biomasa

\section{Destilación}

La ejecución de este proceso se lo realiza mediante la utilización de un alambique de bronce con una capacidad de 5.2 GL, este alambique es proporcionado por la carrera de Ing. Agroindustrial de la facultad "FICAYA" perteneciente a la Universidad Técnica del Norte.

El procedimiento por seguir será el siguiente:

Se vierten 5.2 GL de la substancia previamente fermentada en el interior del alambique.

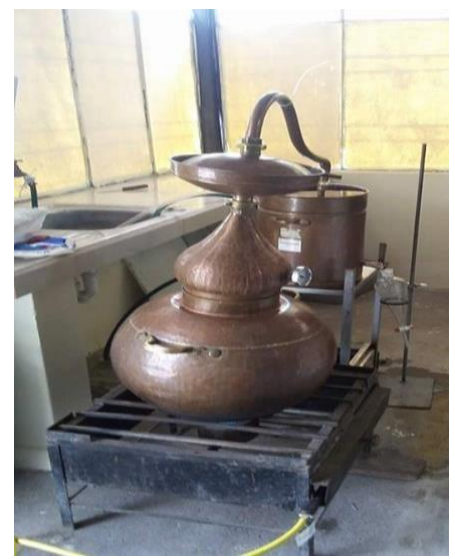

Figura 8. Alambique de 20 Lt.

Se somete a la substancia fermentada a una temperatura establecida en los rangos de 80 ${ }^{\circ} \mathrm{C}$ a $85{ }^{\circ} \mathrm{C}$, provocando así la evaporación y posterior destilación de la mayor parte del etanol presente en el líquido inmerso en el alambique, tras realizar dicho procedimiento y con ayuda de un alcoholímetro se verifica el porcentaje de alcohol presente en la substancia resultante de la destilación, obteniéndose los siguientes datos.

Tabla 8. Resultado de destilación

\begin{tabular}{llll}
\hline Destilación & & & \\
\hline Cantidad de & Temperatura de & Cantidad de & \% de Alcohol (Grados \\
substancia a destilar & destilación $\left({ }^{\circ} \mathrm{C}\right)$ & $\begin{array}{l}\text { Etanol obtenido } \\
\text { (GL) }\end{array}$ & Gay- Lussac) presentes. \\
$\mathbf{1 0 . 5 8}$ & $\mathbf{8 0}^{\circ}-\mathbf{8 5}^{\circ}$ & $\mathbf{3 . 8 3}$ & $\mathbf{3 0}$ \\
\hline
\end{tabular}


Debido tanto a la baja cantidad de etanol obtenido, como también al bajo porcentaje de alcohol presente en el mismo tras la primera destilación, se opta por someter al resultado de la destilación a una serie de rectificaciones con la finalidad de aumentar su porcentaje de alcohol, esta vez se lo realizara en un alambique de bronce con una capacidad de 2.10 GL con rangos de temperatura comprendidos entre 70 y $80^{\circ} \mathrm{C}$, teniendo así el siguiente resultado.

Tabla 9. Resultado de rectificación

\begin{tabular}{llll}
\hline Rectificación de Etanol & & & \\
\hline Cantidad de & Número de & Temperatura de & Cantidad de \\
substancia a destilar & Rectificaciones & destilación $\left({ }^{\circ} \mathrm{C}\right)$ & $\begin{array}{l}\text { Etanol } \\
\text { obtenido (GL) } \\
\text { (GL) }\end{array}$ \\
$\mathbf{3 . 8 3}$ & 3 & $70^{\circ}-80^{\circ}$ & 0.22 \\
\hline
\end{tabular}

\section{Caracterización la muestra de biocombustible obtenido en proporción E10.}

Con la finalidad de conocer las características fisicoquímicas del biocombustible como producto final del presente proyecto, se mezcla gasolina Súper de 92 octanos y el bioetanol obtenido de $96.5 \%$ de alcohol, de la siguiente manera.

\section{Resultados y discusión}

Estimación del consumo de combustible fósil (Gasolinas) en la provincia de ImbaburaEcuador.

Tabla 10. Consumo total de gasolina Extra y Súper en la provincia de Imbabura

\begin{tabular}{lll}
\hline $\begin{array}{l}\text { Tipo de } \\
\text { Gasolina }\end{array}$ & $\begin{array}{l}\text { Población (Miles de } \\
\text { habitantes) }\end{array}$ & $\begin{array}{l}\text { Volumen (Millones de } \\
\text { Galones) }\end{array}$ \\
\hline Extra & 452 & 27.30 \\
Súper & 452 & 3.48 \\
Millones de Galones totales consumidos & $\mathbf{3 0 . 7 8}$ \\
\hline
\end{tabular}

Fuente: (AIHE, 2017).

Se determina que, para el ámbito Automotriz, en la provincia de Imbabura durante el año 2016, el consumo de gasolinas extra y súper asciende a 27.30 y 3.78 millones de galones respectivamente, dando un total de 30.78 millones de galones interpretados como consumo o demanda a nivel provincial.

Determinación las zonas de producción de materia prima en la provincia de Imbabura.

Tabla 11. Producción de Materia Prima en la Provincia

\begin{tabular}{lll}
\hline $\begin{array}{l}\text { Ha Totales } \\
\text { cosechadas }\end{array}$ & $\begin{array}{l}\text { Kg de materia } \\
\text { prima por Ha }\end{array}$ & $\begin{array}{l}\text { Kg Totales de } \\
\text { materia prima }\end{array}$ \\
\hline 1844 & 89363 & $164,785,372$ \\
\hline
\end{tabular}


Se conoce que se pueden producir $89363 \mathrm{Kg}$ de materia prima (BRA) por cada hectárea de maíz cosechada, por consiguiente, se estima que la producción de (BRA) en libras utilizando las 1844 hectáreas registradas como productoras en la provincia, producen 164,785,372 de Kg totales aprovechables

Tabla 12. Método para tratar la biomasa residual del maíz

\begin{tabular}{lllll}
\hline Método & $\begin{array}{l}\text { Tiempo de } \\
\text { Ejecución }\end{array}$ & $\begin{array}{l}\text { Costo } \\
(\$)\end{array}$ & $\begin{array}{l}\text { Cantidad } \\
(\mathrm{gr})\end{array}$ & $\begin{array}{l}\text { Tiempo de Adquisición del } \\
\text { producto }\end{array}$ \\
\hline $\begin{array}{l}\text { Enzimas } \\
\text { (DXilosa) }\end{array}$ & $\begin{array}{l}\text { 8 días de } \\
\text { Incubación }\end{array}$ & $\mathbf{2 5 0}$ & $\mathbf{1 0}$ & $\mathbf{1}$ semana \\
\hline
\end{tabular}

Se establece que el mejor método para tratar los despojos de maíz (BRA) y producir el resultado deseado (liberación de azucares) es por medio de la enzima D-Xilosa, teniendo un lapso de incubación de 8 días y una demora de adquisición de dicho producto de una semana que es considerablemente factible conforme al desarrollo del presente proyecto. (Pereira, 1957). Producir Bioetanol en base a despojos de maíz.

Tabla 13. Datos del Bioetanol resultante final

\begin{tabular}{|c|c|c|c|}
\hline \multicolumn{4}{|l|}{ Bioetanol Obtenido } \\
\hline Característica & Unidad & $\begin{array}{l}\text { Datos } \\
\text { obtenidos }\end{array}$ & $\begin{array}{l}\text { Datos } \\
\text { normativa NTE- INEN } 2478 \\
\text { (Mínimos/Máximos) }\end{array}$ \\
\hline $\begin{array}{l}\text { Cantidad de bioetanol } \\
\text { Obtenido }\end{array}$ & $(\mathrm{GL})$ & 0.22 & ---------- \\
\hline \% de Alcohol presente & $\begin{array}{l}\left({ }^{\circ} \mathrm{Gay}\right. \\
\text { Lussac) }\end{array}$ & $96.5^{\circ}$ & $96.3 /----$ \\
\hline PH & ----- & 6.8 & $6.5 / 9.0$ \\
\hline Coloración & ----- & Incoloro & --------- \\
\hline Olor & ----- & Característico & ---------- \\
\hline Densidad & $\mathrm{Kg} / \mathrm{m}^{3}$ & 0,768 & $----/ 791,5$ \\
\hline
\end{tabular}

De acuerdo con los datos mostrados en la presente tabla se tiene que los mismos están dentro de los rangos (Límites) que la normativa NTE-INEN 2478 para etanol anhidro grado carburante desnaturalizado, cabe recalcar que dichos datos fueron obtenidos de acuerdo con los equipos proporcionados por la carrera de Ingeniería Agroindustrial perteneciente a la Universidad Técnica del Norte.

Tabla 14. Balance general de resultados obtenidos

\begin{tabular}{lllll}
\hline $\begin{array}{l}\text { Kg de } \\
\text { Biomasa } \\
\text { Utilizada }\end{array}$ & $\begin{array}{l}\text { GL de } \\
\text { substancia } \\
\text { fermentada base } \\
\text { Obtenida }\end{array}$ & $\begin{array}{l}\text { Número de } \\
\text { Rectificaciones }\end{array}$ & $\begin{array}{l}\text { Cantidad de } \\
\text { Etanol } \\
\text { Obtenido } \\
(\text { GL })\end{array}$ & $\begin{array}{l}\text { \% Alcohol } \\
\left({ }^{\circ} \text { Gay Lussac }\right) \\
\text { presente }\end{array}$ \\
\hline $\mathbf{1 1 . 3 6}$ & $\mathbf{1 0 . 5 8}$ & $\mathbf{4}$ & $\mathbf{0 . 2 2}$ & $\mathbf{9 6 . 5}$ \\
\hline
\end{tabular}


Al tener un balance general de resultados obtenidos referente al rendimiento Biomasa/Etanol se comprende que por cada $11.36 \mathrm{Kg}$ de (BRA) empleados tendremos 0,22 Gl de bioetanol con un porcentaje de alcohol presente del 96,5\% tras realizar 4 rectificaciones (destilaciones).

Tabla 15. Estimación de Producción de Bioetanol

\begin{tabular}{lll}
\hline $\begin{array}{l}\text { Kg Obtenidos } \\
\text { por Ha }\end{array}$ & $\begin{array}{l}\text { Ha Totales } \\
\text { Disponibles }\end{array}$ & $\begin{array}{l}\text { GL Totales Obtenidos } \\
\text { de Bioetanol }\end{array}$ \\
\hline 1768 & 1844 & $3,260,855$ \\
\hline
\end{tabular}

Se tiene una estimación de producción de bioetanol basada en la relación de las hectáreas totales de producción registradas en la provincia de Imbabura $(1,844 \mathrm{Ha})$ y los litros producidos por hectárea empleada, teniendo de esta manera una proyección de 3, 260,855 de GL de bioetanol producidos. Comparación de datos obtenidos de caracterización de biocombustible y la norma ASTM 910-02.

Tabla 16. Comparación de datos norma ASTM

\begin{tabular}{|c|c|c|c|c|c|c|}
\hline \multicolumn{5}{|c|}{ Datos obtenidos biocombustible e10 } & \multicolumn{2}{|c|}{$\begin{array}{l}\text { Norma ASTM 910- } \\
02\end{array}$} \\
\hline ENSAYO & & $\begin{array}{l}\text { Norma } \\
\text { método }\end{array}$ & Unidad & Valor obtenido & Mínimo & Máximo \\
\hline \multicolumn{2}{|c|}{ Número de Octano (RON) } & NTE INEN & ----- & 96.3 & 98 & ---- \\
\hline Ensayo de & Temperatura al & ASTM & ${ }^{\circ} \mathrm{C}$ & 52.1 & ----- & 75 \\
\hline \multirow[t]{4}{*}{ Destilación } & Temperatura al & D86-15 & ${ }^{\circ} \mathrm{C}$ & 110.5 & ----- & 105 \\
\hline & $\begin{array}{l}\text { Temperatura al } \\
90 \%\end{array}$ & & ${ }^{\circ} \mathrm{C}$ & $\begin{array}{l}\text { No alcanzó } \\
\text { elporcentaje }\end{array}$ & ----- & 135 \\
\hline & Punto Final & & ${ }^{\circ} \mathrm{C}$ & 170 & ----- & 170 \\
\hline & Residuo & & $\%$ & 1.0 & ----- & 1.5 \\
\hline \multicolumn{2}{|c|}{ Presión de vapor Reid } & $\begin{array}{l}\text { ASTM } \\
\text { D323-15A }\end{array}$ & $\mathrm{kPa}$ & 58.6 & 38 & 49 \\
\hline \multicolumn{2}{|c|}{ Corrosión a lámina de cobre } & $\begin{array}{l}\text { ASTM } \\
\text { D130-12 }\end{array}$ & ----- & $1 \mathrm{~A}$ & ----- & 1 \\
\hline \multicolumn{2}{|c|}{ Contenido de azufre } & $\begin{array}{l}\text { ASTM } \\
\text { D4294-16 }\end{array}$ & $\%$ & 0,02 & ---- & 0,065 \\
\hline \multicolumn{2}{|c|}{ Contenido de gomas } & $\begin{array}{l}\text { ASTM } \\
\text { D381-12 }\end{array}$ & $\begin{array}{l}\mathrm{Mg} / 100 \\
\mathrm{~mL}\end{array}$ & 0,2 & ---- & 0.05 \\
\hline
\end{tabular}

Fuente: (RTCA, 2006).

Comparación de datos obtenidos de caracterización de biocombustible y norma RTCA $169-2006$.

Tabla 17. Comparación de datos norma RTCA

\begin{tabular}{|c|c|c|c|c|}
\hline DATOS O & USTIBLE & & & A 169 \\
\hline ENSAYO & $\begin{array}{l}\text { NORMA- } \\
\text { MÉTODO }\end{array}$ & UNIDAD & $\begin{array}{l}\text { VALOR } \\
\text { OBTENIDO }\end{array}$ & Máximo \\
\hline
\end{tabular}




\begin{tabular}{|c|c|c|c|c|c|c|}
\hline \multicolumn{2}{|c|}{ Número de Octano (RON) } & NTEINEN & $\begin{array}{ll}--- \\
\end{array}$ & 96.3 & 83 & 88 \\
\hline \multirow{5}{*}{$\begin{array}{l}\text { Ensayo de } \\
\text { Destilación }\end{array}$} & Temperatura al & $\begin{array}{l}2102 \\
\text { ASTM D86- }\end{array}$ & ${ }^{\circ} \mathrm{C}$ & 52.1 & 77 & 121 \\
\hline & $\begin{array}{l}10 \% \\
\text { Temperatura al }\end{array}$ & 15 & ${ }^{\circ} \mathrm{C}$ & 110.5 & ----- & 105 \\
\hline & $\begin{array}{l}50 \% \\
\text { Temperatura al } \\
90 \%\end{array}$ & & ${ }^{\circ} \mathrm{C}$ & $\begin{array}{l}\text { No Alcanzó el } \\
\text { porcentaje }\end{array}$ & ----- & 190 \\
\hline & Punto Final & & ${ }^{\circ} \mathrm{C}$ & 170 & ----- & 225 \\
\hline & Residuo & & $\%$ & 1.0 & ----- & 2 \\
\hline \multirow{2}{*}{\multicolumn{2}{|c|}{$\begin{array}{l}\text { Presión de vapor Reid } \\
\text { Corrosión a la lámina de cobre }\end{array}$}} & ASTM D323- & $\mathrm{kPa}$ & 58.6 & 38 & 69 \\
\hline & & $\begin{array}{l}\text { ASTM D130- } \\
12\end{array}$ & ----- & $1 \mathrm{~A}$ & ------ & 1 \\
\hline \multirow{2}{*}{\multicolumn{2}{|c|}{$\begin{array}{l}\text { Contenido de azufre } \\
\text { Contenido de gomas }\end{array}$}} & ASTM & & 0,020 & ----- & 0,10 \\
\hline & & ASTM D381- & $\mathrm{Mg} / \mathbf{1 0 0}$ & 0,2 & ...-. & 4 \\
\hline
\end{tabular}

El biocombustible en proporción E10 conformado por gasolina de 92 octanos y Bioetanol de $96.3 \%$ de contenido de alcohol, tras realizar su caracterización, se establece que no cumple con las siguientes especificaciones establecidas en la norma RTCA 169 -2006 para requerimientos de gasolinas.

Tabla 18. Análisis de datos

\begin{tabular}{lll}
\hline Especificaciones & Cumple & No Cumple \\
\hline Número de Octano & $\mathrm{X}$ \\
Destilación a 10\%,50\%, $90 \%$ & $\mathrm{X}$ \\
Punto final & $\mathrm{X}$ \\
Residuo & $\mathrm{X}$ \\
Presión vapor Reid & $\mathrm{X}$ \\
Corrosión a la lámina de cobre & $\mathrm{X}$ & \\
Contenido de Azufre & & $\mathrm{X}$ \\
Contenido de Gomas & $\mathrm{X}$ \\
\hline
\end{tabular}

Comparación de datos obtenidos de caracterización de biocombustible y norma NTEINEN 935.

Tabla 19. Comparación de datos norma NTE -INEN 935

\begin{tabular}{lllllll}
\hline \multicolumn{2}{l}{ DATOS OBTENIDOS BIOCOMBUSTIBLE E10 } & & \multicolumn{4}{l}{ Norma NTE-INEN 935 } \\
\hline ENSAYO & $\begin{array}{l}\text { NORMA } \\
\text { MÉTODO }\end{array}$ & UNIDAD & VALOR & Mínimo & Máximo \\
OBTENIDO & & & \\
\multicolumn{2}{l}{$\begin{array}{l}\text { Número de Octano (RON) } \\
\text { Ensayo de }\end{array}$} & NTE INEN & ----- & 96.3 & 92.0 & ---- \\
Destilación & Temperatura al & & ${ }^{\circ} \mathrm{C}$ & 52.1 & ---- & $\mathbf{7 0}$ \\
& Temperatura al & & ${ }^{\circ} \mathrm{C}$ & 110.5 & ---- & $\mathbf{1 2 1}$ \\
& Temperatura al & ASTM D86- & ${ }^{\circ} \mathrm{C}$ & No alcanzó el & ---- & $\mathbf{1 9 0}$ \\
& $90 \%$ & 15 & & porcentaje & & \\
& Punto Final & & ${ }^{\circ} \mathrm{C}$ & 170 & ---- & $\mathbf{2 2 0}$ \\
\hline
\end{tabular}




\begin{tabular}{llllll}
\hline \multicolumn{1}{c}{ Residuo } & & $\%$ & 1.0 & ----- & $\mathbf{2}$ \\
Presión de vapor Reid & ASTM D323- & $\mathrm{kPa}$ & 58.6 & ---- & $\mathbf{6 0}$ \\
Corrosión a la lámina de cobre & ASTM D130- & ----- & $1 \mathrm{~A}$ & ---- & $\mathbf{1}$ \\
Contenido de azufre & ASTM & $\mathbf{\%}$ & $\mathbf{0 , 0 2 0}$ & ---- & $\mathbf{0 , 0 6 5}$ \\
\hline
\end{tabular}

Conforme los datos detallados mostrados en esta tabla, mismos que fueron obtenidos posterior a la caracterización (Análisis Fisicoquímico) a la que fue sometida la muestra (Biocombustible E10) enviada al laboratorio de petróleos de Ingeniería Química de la EPN, señalan que el antes mencionado si cumple con todas las especificaciones establecidas en la normativa NTE-INEN 935 para requerimientos de gasolinas, consecuentemente se tiene un análisis comparativo de cada uno de los datos obtenidos.

Existe una diferencia de datos referentes a temperaturas de destilación, existiendo una disminución en las registradas por el biocombustible E10 con respecto a las de la gasolina Súper, se tiene el siguiente análisis de datos.

Temperatura al $10 \%$

La disminución de temperatura registrada por el biocombustible E10 al $10 \%$ de temperatura es de $-17.9^{\circ} \mathrm{C}$ de esta manera se constata una mayor capacidad para facilitar el encendido en frio del motor o a bajas temperaturas por parte de este combustible.

Temperatura al $50 \%$

La disminución de temperatura registrada por el biocombustible E10 al $50 \%$ de temperatura es de $-10.5^{\circ} \mathrm{C}$ evidenciándose su capacidad para alcanzar temperaturas acordes a la del funcionamiento del motor, desempeñándose de mejor manera a temperaturas bajas.

Temperatura al $90 \%$

Para este porcentaje de temperatura los datos de destilación registrados por el biocombustible no alcanzaron los valores legibles por el equipo de análisis, lo que evidencia su alto valor de vaporización.

-Presión de Vapor Reid

Tabla 20. Comparación de datos de Vapor Reid

\begin{tabular}{ll}
\hline $\begin{array}{l}\text { Gasolina Súper (Norma } \\
\text { INEN-935) }\end{array}$ & $\begin{array}{l}\text { Biocombustible } \\
\text { E10 }\end{array}$ \\
\hline $60 \mathrm{KPa}$ & $58.6 \mathrm{KPa}$ \\
\hline
\end{tabular}

Se tiene una disminución de los valores registrados por el biocombustible de $1.4 \mathrm{KPa}$ en relación a la gasolina súper, considerándose a esta diferencia de valores como despreciable, asumiéndose que tiene el mismo valor de evaporación que la gasolina además de comportarse de la igual manera al ser almacenados y utilizados bajo una temperatura de $100^{\circ} \mathrm{F}$.

Corrosión a la Lámina de Cobre (Gallina, (2010) 
Tabla 21. Comparación de datos de Corrosión a la lámina de cobre

\begin{tabular}{ll}
\hline $\begin{array}{l}\text { Gasolina Súper } \\
\text { (Norma INEN-935) }\end{array}$ & $\begin{array}{l}\text { Biocombustible } \\
\text { E10 }\end{array}$ \\
\hline $1 \mathrm{~A}$ & $1 \mathrm{~A}$ \\
\hline
\end{tabular}

Los datos registrados por el biocombustible en este ensayo no presentan variaciones en relación al registrado por la gasolina Súper, asumiéndose de esta manera que los dos tipos de combustibles poseen la misma capacidad corrosiva hacía varias aleaciones del motor de combustión interna.

-Contenido de Azufre

Tabla 22. Comparación de datos de Contenido de Azufre

\begin{tabular}{ll}
\hline $\begin{array}{l}\text { Gasolina Súper } \\
\text { (Norma INEN-935) }\end{array}$ & $\begin{array}{l}\text { Biocombustible } \\
\text { E10 }\end{array}$ \\
\hline $0.065 \%$ & $0.020 \%$ \\
\hline
\end{tabular}

De acuerdo con los datos registrados, se establece una disminución de la cantidad de azufre presente en el biocombustible del $0.035 \%$ en relación con el valor registrado por la gasolina Súper, señalando de igual manera que el biocombustible es menos propenso a corroer las diferentes aleaciones del motor de combustión interna, a influir en el mal funcionamiento del catalizador y además disminuye las emisiones de NOx que produce al ser combustionado.

-Contenido de Gomas

Tabla 23. Comparación de datos de Contenido de Gomas

\begin{tabular}{ll}
\hline $\begin{array}{l}\text { Comparación del valor de contenido de } \\
\text { Gomas }\end{array}$ & \\
\hline $\begin{array}{l}\text { Gasolina Súper } \\
\text { (Norma INEN-935) }\end{array}$ & Biocombustible \\
E10 \\
$4.0 \mathrm{mg} / 100 \mathrm{ml}$ & $0.2 \mathrm{mg} / 100 \mathrm{ml}$ \\
\hline
\end{tabular}

Se tiene una diferencia considerable de datos obtenidos en este ensayo, teniendo como resultado del análisis del biocombustible una disminución de - $3.8 \mathrm{mg} / 100 \mathrm{ml}$ en relación con la gasolina súper, asumiendo de esta manera un bajo potencial en el biocombustible para impregnarse en las paredes internas del motor, impidiendo de esta manera el desarrollo a largo plazo de una alteración en la relación de compresión del motor

\section{Consideraciones generales}

Para este punto se considera la cantidad de agua presente en el biocombustible al estar compuesto por bioetanol que no es totalmente deshidratado, además de sus repercusiones en el 
motor al ser combustionado y utilizado a largo plazo, tomando como una alternativa la realización de modificaciones a un motor para disminuir futuros problemas en el mismo.

Tabla 24. Modificaciones considerables al usar el biocombustible E10

\begin{tabular}{|c|c|}
\hline \multicolumn{2}{|l|}{ Uso de Bioetanol } \\
\hline $\begin{array}{lll}\text { Cantidad de } & \text { bioetanol } \\
\text { presente } & & \\
\end{array}$ & $\begin{array}{l}\text { Modificaciones } \\
\text { realizarse }\end{array}$ \\
\hline \multirow[t]{5}{*}{$\leq 10 \%$} & Sistema de Inyección \\
\hline & Bomba de Combustible \\
\hline & Filtro de Combustible \\
\hline & Catalizador \\
\hline & Sistema de Escape \\
\hline
\end{tabular}

Es importante mencionar que las modificaciones mostradas en la tabla número 29 son aplicables para vehículos que utilicen de manera prolongada este tipo de biocombustible en la mencionada proporción y además que tengan de 15 a 20 años de antigüedad (Galante-Fox, 2007)

\section{Conclusiones}

El consumo de gasolinas (Extra de 88 Octanos RON y Súper de 92 Octanos RON) en la provincia de Imbabura representa solo el 2,68 \% del consumo anual a nivel nacional. De las zonas registradas por el "MAGAP" como productoras del Chaucho Mejorado en la Provincia de Imbabura, el cantón Otavalo es el mayor productor de dicha materia prima, representando al $44 \%$ de la producción total de la misma.

El acceso a enzimas y reactivos químicos apropiados o a fines para el desarrollo del presente proyecto es muy limitado, optándose por esta razón por la importación del reactivo a utilizar (D-Xilosa) en proporciones de 0.70 gr por cada $\mathrm{Kg}$ de biomasa utilizado.

El porcentaje más alto de Alcohol $\left({ }^{\circ} \mathrm{Gay}\right.$ Lusaac) que se pudo obtener al momento de llevar a cabo la destilación del bioetanol es del 96.5\%, los datos del bioetanol obtenido si están dentro de los parámetros establecidos por la normativa NTE- INEN 2478 para Etanol anhidro grado carburante desnaturalizado.

Conociendo que se puede producir 1,768 gal de bioetanol por Ha de maíz utilizada, se estima que si emplea las 1,844 Ha de maíz (Chaucho Mejorado) producidas en la Provincia de Imbabura se podría producir 3,260.855 GL de Bioetanol, satisfaciendo de esta manera los escenarios E5 y E10 de consumo de combustible fósil (Gasolinas) a nivel provincial en el año 2016.

La mezcla del Bioetanol producido (de $96.5 \%$ de Alcohol) en adición a la gasolina Súper (de 92 Octanos RON (Wuithier, 1971)) conforman un Biocombustible en proporción E10, el mismo que mediante los resultados obtenidos posterior a su caracterización, se establece que si cumple con la normativa (INEN, 2017)para requerimientos de Gasolinas. 
De los resultados obtenidos tras la caracterización del biocombustible en proporción E10, se establece que la diferencia más notable de los mismos corresponden al número de Octano con 4.3 puntos de diferencia positiva, Contenido de azufre con $0.035 \%$ de diferencia (disminución)y contenido de gomas con $3.8 \mathrm{mg} / 100 \mathrm{ml}$ de diferencia (disminución), en relación a los datos registrados por la gasolina súper establecidos en la NTE - INEN 935 (INEN, 2017)para requerimientos de Gasolinas, en base a estos datos se establece una mejora en cuanto al rendimiento del combustible, potencia del motor, cantidad de NOx emanado tras la combustión y la ausencia de sedimentos producidos por el aprovechamiento del biocombustible a largo plazo obtenidas de los datos obtenidos.

\section{Bibliografía}

AIHE. (5 de abril de 2017). Petróleo en cifras, AIHE, Petróleo en cifras, págs. 16-29.

Dias, M. O. (2011). Simulation of integrated first and second generation bioethanol production from sugarcane: comparison between different biomass pretreatment methods. Journal of Industrial Microbiology $1 \&$ Biotechnology, 955--966.

Elfasakhany, A. (Volume 139). Engine performance evaluation and pollutant emissions analysis using ternary bio-ethanol-iso-butanol-gasoline blends in gasoline engines. In Journal of Cleaner Production, 1057-1067.

Espinosa Cajas, F. J. (2013). Obtención de etanol mediante hidrólisis alcalina, enzimática y fermentación a partir del excedente orgánico del banano variedad Musa Paradisíaca. Tesis de Grado para la obtención del Título de Ingeniero Químico. Quito: Carrera de Ingeniería Química. Quito: UCE. 101 p.

Galante-Fox, R. J. (2007). Controlling Induction System Deposits in Flexible Fuel Vehicles Operating on E85. SAE Technical Paper.

Gallina, A. L. ((2010). A corrosão do aço inoxidável austenítico 304 em biodiesel. Revista Escola de Minas, 63(1), 71-75.

INEN.

(2017). http://www.normalizacion.gob.ec/wpcontent/uploads/downloads/2015/10/nte_inen_935_9r.pdf. Obtenido de http://www.normalizacion.gob.ec/wpcontent/uploads/downloads/2015/10/nte_inen_935_9r.pdf.

MAGAP-DPA UZI. (2016). MAGAP-DPA UZI, . IMBABURA: MAGAP.

Naz, M. A. (2014). Global estimates of energy consumption and greenhouse gas emissions. Renewable and Sustainable Energy Reviews, 336 - 344.

OPEP. (2017). https://asb.opec.org/. Obtenido de https://asb.opec.org/. 
Pereira, J. N. (1957). Nutrition And Physiology Of Pseudomonas Fragi. 74(6), . Journal of Bacteriology, 710-713.

Prefectura de Imbabura. (2016). Datos generales. Ibarra: Prefectura Imbabura.

RTCA. (2006). http://standards.globalspec.com/stds/sdo/rtca. Obtenido de IEEE.

Sánchez Riaño, A. M., \& Gutiérrez Morales, A. M. (2010). Producción de bioetanol a partir de subproductos. Revista Tumbaga , 61-91.

Wuithier, P. .. (1971). El petróleo. Refino y Traramiento químico. Tomo I. En P. Wuithier, El petróleo. Refino y Traramiento químico. Tomo I (pág. 15.). Ediciones CEPSA, S.A. .

Yánez G., C. (2013). Ficha técnica del “Chaucho Mejorado. Quito, Ecuador: INIAP, Estación Experimental Santa Catalina: INIAP-122. 